\title{
Metodología para la Cuantización de Campos de Yang-Mills
}

\author{
Pedro Romano-Aportela $^{(1)}$, Martín Salazar-Pereyra ${ }^{(1)}$, Claudia Lozano-Mora ${ }^{(1)}$ y \\ Raúl Lugo-Leyte ${ }^{(2)}$ \\ (1) Tecnológico de Estudios Superiores de Ecatepec, División de Ingeniería Mecatrónica e \\ Industrial, Avda. Tecnológico, Col. Valle de Anáhuac, 55210, Ecatepec de Morelos, \\ Estado de México-México (e-mail: pedroromano@hotmail.com). \\ (2) Univ. Autónoma Metropolitana-Iztapalapa, Departamento de Ingeniería de Procesos e \\ Hidráulica, Avda. San Rafael Atlixco No. 186, Col. Vicentina, Iztapalapa, 09340, \\ México, D.F.-México (e-mail: lulr@xanum.uam.mx).
}

Recibido Dic. 03, 2010; Aceptado Ene. 24, 2011; Versión Final Recibida Abr. 06, 2011

\begin{abstract}
Resumen
Se analizan las interacciones electromagnéticas y nucleares débiles utilizando el principio fundamental de simetría en espacios abstractos denominados teoría de campos de Yang-Mills, también conocidos como campos de norma (gauge fields) y el mecanismo de Higgs. Los campos de norma actúan como mediadores de las interacciones, cuyo alcance está determinado de manera directa por la masa. Por este motivo los campos de norma se unen al mecanismo de Higgs que genera masa a los portadores de las interacciones, manteniendo la teoría invariante bajo una transformación de norma. Esto se logra a través de un rompimiento espontaneo de simetría para finalmente aplicar esta metodología con la finalidad de unificar las teorías de las interacciones considerando el modelo estándar de Weinberg-Salam.
\end{abstract}

Palabras clave: campos de norma, campos de Yang-Mills, bosones de Higgs, bosones de Goldstone, rompimiento espontaneo de simetría.

\section{Methodology for the Quantization of Yang-Mills Fields}

\begin{abstract}
The electromagnetic and weak nuclear interactions are analyzed using the fundamental principle of symmetry in abstract spaces named theory of Yang-Mills fields, also known as gauge fields, and Higgs's mechanism. Gauge fields are mediators of interactions, whose scope is determined directly by the mass. For this reason, gauge fields are joined with the Higgs mechanism that generates mass to the interaction carriers, maintaining the invariant theory under a gauge transformation. This is achieved through spontaneous symmetry breaking to finally applying this methodology in order to unify the theories of interactions considering the Weinberg-Salam standard model.
\end{abstract}

Keywords: gauge fields, Yang-Mills fields, Higgs bosons, Goldstone bosons, spontaneous symmetry breaking. 


\section{INTRODUCCIÓN}

Los progresos en la teoría cuántica de campos durante los últimos años se han visto fuertemente impulsados debido al desarrollo de la teoría cuántica de campos de Yang - Mills, llamados también campos de norma (Abers, et al., 1973). Estos campos presentan nuevas posibilidades para la descripción de las interacciones de las partículas elementales en el marco de la teoría cuántica de campos (Halzen, et al., 1984). De hecho los campos de norma están involucrados en casi todos los modelos de las interacciones: nuclear fuerte, nuclear débil y electromagnética (Schweber, 2005). También esta teoría se aplica para la unificación de todas las interacciones en una sola interacción fundamental, además de que todas las fuerzas de la naturaleza se rigen por un principio de simetría fundamental, que es la invariancia de norma (thooft, 2007). Esto se deriva en los siguientes hechos:

1.- La electrodinámica cuántica es la única teoría completamente confirmada experimentalmente y la cual es un caso particular de la teoría de norma, donde el portador de la interacción es el fotón el cual tiene masa nula y de aquí que su alcance sea infinito (Itzykson, et al., 2005)

2.- La interacción nuclear débil también se describe de manera consistente en el marco de las teorías de norma, donde los portadores de la interacción son los bosones vectoriales intermedios de espín uno, conocidos como $\left\{W^{ \pm}, Z^{0}\right\}$ con masas muy grandes de aproximadamente 80 Gev (Giga electronvolts), por tal razón está interacción es de muy corto alcance. (Georgi, 2009)

3. El boson $Z^{0}$ conocido como la corriente neutra, fue descubierto experimentalmente en el Centro Europeo de Investigaciones Nucleares (CERN) en 1984. Asimismo, la teoría de norma unifica las interacciones débiles y electromagnéticas, conocida como la teoría electrodébil o el modelo estándar de Weinberg - Salam, este modelo está basado en el grupo de simetría $S U(2) \times U(1)$. La unificación de estas dos interacciones con mediadores de masas diferentes se realiza a través de de un rompimiento espontaneo de simetría y el mecanismo de Higgs. (Taylor, 1976)

4-. Los modelos fenomenológicos de quarks de las interacciones fuertes también tienen su fundamentación natural en el marco de las teorías de norma (Bjorken. et al. 1986)

5.- La interacción gravitacional también puede ser colocada en este esquema. La teoría del campo unificado de Einstein, resurge y se refuerza como una teoría de norma. (Kiefer, 2007). La teoría de Yang - Mills es esencial en la física (Vacaru, 2005) y ocupa un lugar importante en el futuro de la teoría de partículas elementales (Damour. Et al. 2008).

\section{TEORÍAS DE NORMA}

Es de gran utilidad cuando se considera un sistema físico obtener propiedades bien definidas que a su vez caractericen o etiqueten al sistema en sí, por ejemplo las cantidades que no cambian con el tiempo o que permanecen constantes y que por lo tanto llevan asociadas una ley de conservación. Estas se les conocen como propiedades de simetría del sistema (Bjorken, et al., 1986).

En la física no - relativista, son bien conocidas las leyes de conservación provenientes de la homogeneidad e isotropía del espacio y del tiempo absoluto; se obtiene así, la conservación del momento lineal, del momento angular y de la energía, respectivamente (Schweber, 1961).

En general el teorema de Noether establece que para cualquier transformación continua de simetría bajo la cual la lagrangiana permanezca invariante implica la existencia de una corriente conservada (Schweber, 2005).

$$
\partial^{\mu} J_{\mu}(x)=0
$$


Con una carga definida por:

$Q=\int d^{3} x J_{0}(x)$

Por ejemplo, para una transformación infinitesimal

$$
\phi(x) \rightarrow \phi^{\prime}(x)=\phi(x)+\delta \phi(x)
$$

Se tiene

$$
\delta L=\frac{\delta L}{\delta \varphi} \delta \varphi+\frac{\delta L}{\delta\left(\partial_{\mu} \varphi\right)} \delta\left(\partial_{\mu} \varphi\right)
$$

y por las ecuaciones de Euler - Lagrange:

$\partial_{\mu}\left[\frac{\delta L}{\delta\left(\partial_{\mu} \varphi\right)}\right]-\frac{\delta L}{\delta \varphi}=0$

$\delta L=\partial_{\mu}\left[\frac{\delta L}{\delta\left(\partial_{\mu} \varphi\right)}\right] \delta \varphi+\frac{\delta L}{\delta\left(\partial_{\mu} \varphi\right)} \partial_{\mu}(\delta \varphi)=\partial_{\mu}\left[\frac{\delta L}{\delta\left(\partial_{\mu} \varphi\right)} \delta \varphi\right]$

Entonces se tiene simetría interna si

$\delta L=0$

Esto es:

$J_{\mu}=\frac{\delta L}{\delta\left(\partial_{\mu} \phi\right)} \delta \phi$

Escribiendo a $\delta \phi$ como:

$\delta \phi_{i}=i \varepsilon^{a} t^{a}{ }_{i j} \phi_{j}$

Si los parámetros $\varepsilon^{a}$ son independientes de $x$, la transformación se dice global (o de primera clase), pues los campos se transforman de la misma manera para dos puntos arbitrariamente separados (Weyl, 1950). Si en cambio $\varepsilon^{a}=\varepsilon^{a}(x)$ la transformación es local (segunda clase). $t^{a}$ son un conjunto de matrices que satisfacen:

$\left[t^{a}, t^{b}\right]=i c^{a b c} t^{c}$

El ejemplo más simple de simetría global es el grupo Abeliano $U(1)$, en donde la carga eléctrica se conserva. El caso de simetría global no - abeliana más simple es el grupo SU(2) donde se conserva el espín isotópico (Catren, 2008).

TRANSFORMACIONES DE NORMA LOCAL. GRUPO U(1) LOCAL

La electrodinámica posee más simetrías que las transformaciones de norma global, por ejemplo; las transformaciones que dependen del espacio - tiempo.

$\phi(x) \rightarrow \phi^{\prime}(x)=\exp \{-i q \theta(x)\} \phi(x)$ 
Donde $q$ es el eigenvalor del grupo y $\theta=\theta(x)$. Por lo tanto, en forma diferencial:

$$
\delta \phi=\phi^{\prime}-\phi=-i q \theta(x) \phi(x)
$$

Los términos de la lagrangiana que dependen sólo de $\phi$ son invariantes bajo (9), pero no aquellos que contienen los gradientes de los campos, tales como la energía cinética, esto es

$\partial_{\mu} \phi \rightarrow \partial_{\mu} \phi^{\prime}=\exp \{-i q \theta(x)\} \partial_{\mu} \phi-i q\left[\partial_{\mu} \theta(x)\right] \exp \{-i q \theta(x)\} \phi(x)$

Por el principio de acoplamiento mínimo se tiene (Leite, 1977):

$\partial_{\mu} \rightarrow \partial_{\mu}-i e q A_{\mu}$

En donde $A_{\mu}$ es el campo del fotón o campo de norma.

Con base en lo anterior se define la derivada covariante como:

$D_{\mu} \equiv \partial_{\mu}-i e q A_{\mu}$

donde e es la carga eléctrica del electrón.

Entonces se requiere que $\partial_{\mu} \phi$ se transforme tal que $\left(\partial_{\mu}-i e q A_{\mu}\right) \phi(x)$ se transforme de la misma manera que $\phi$, es decir:

$\left(\partial_{\mu}-i e q A_{\mu}^{\prime}\right) \phi^{\prime}(x)=\exp \{-i q \theta(x)\}\left(\partial_{\mu}-i e q A_{\mu}\right) \phi(x)$

y entonces $L$ será invariante de norma local.

Para que se cumpla (16), $A_{\mu}$ se transforma:

$A_{\mu}^{\prime}=A_{\mu}-\frac{1}{e} \partial_{\mu} \theta(x)$

\section{Esto es}

$\delta A_{\mu}=-\frac{1}{e} \partial_{\mu} \theta(x)$

Ahora para que $A_{\mu}$, pueda ser considerada como una verdadera variable dinámica se debe construir el término de energía cinética y además su término de masa (Halzen, et al., 1984).

El término de energía cinética se construye a partir del tensor de Faraday $F_{\mu \nu}$

$$
F_{\mu v}=\partial_{\mu} A_{v}-\partial_{v} A_{\mu}
$$

El cual es invariante bajo la transformación (17)

Entonces el término de energía cinética es:

$L=-\frac{1}{4} F_{\mu v} F^{\mu v}$ 
El término de masa de $A_{\mu}$ es: $-\frac{1}{2} m^{2} A_{\mu} A^{\mu}$

El cual viola la invariancia de norma local, tal que está invariancia es imposible a menos que el campo de norma $A_{\mu}$ carezca de masa.

De manera análoga se puede tratar el caso en el que $\psi(x)$ sea el campo de un fermión. Entonces, la lagrangiana para (QED) invariante bajo el grupo $U(1)$ es (Bogoliubov, et al., 1959):

$$
L=\bar{\psi} i \gamma^{\mu}\left(\partial_{\mu}-i e A_{\mu}\right) \psi-m \bar{\psi} \psi-\frac{1}{4} F_{\mu \nu} F^{\mu v}
$$

La lagrangiana (21) se caracteriza por:

1) El fotón debe tener masa cero

2) El acoplamiento mínimo del fotón con el campo $\psi$ está contenido en la derivada covariante $D_{\mu} \psi$ la cual puede construirse a partir de las propiedades de transformación de $\psi$ bajo el grupo de simetría. Esto se conoce como el principio de universalidad.

3) No contener términos de auto acoplamiento fotónico, ya que el fotón no tiene carga.

De estas características las dos primeras subsisten en teorías de norma no - abeliana (local), pero no así la última, y por lo tanto estás teorías serán no lineales y las propiedades por lo tanto serán distintas.

\section{SIMETRÍAS DE NORMA NO - ABELIANA}

Sea el campo del fermión un doblete de isoespín

$\psi=\left(\begin{array}{l}\psi_{1} \\ \psi_{2}\end{array}\right)$

El grupo sU(2) es el grupo de transformaciones tales que (Bjorken, et al., 1986):

$\psi(x) \rightarrow \psi^{\prime}(x)=\exp \left\{-i \frac{\vec{\tau} \cdot \vec{\theta}(x)}{2}\right\} \psi(x)$

Donde $\vec{\tau}=\left\{\tau_{1}, \tau_{2}, \tau_{3}\right\}$ son las matrices de Pauli, las cuales obedecen las siguientes relaciones de conmutación (Weyl, 1950)

$\left[\frac{\tau_{i}}{2}, \frac{\tau_{j}}{2}\right]=i \varepsilon_{i j k} \frac{\tau_{k}}{2}, \quad i, j k=1,2,3$

y $\vec{\theta}=\left(\theta_{1}, \theta_{2}, \theta_{3}\right)$ son los parámetros de la transformación $S U(2)$.

La lagrangiana libre es

$L=\bar{\psi}(x)\left(i \gamma^{\mu} \partial_{\mu}-m\right) \psi(x)$

La cual es invariante bajo la transformación de norma global, sin embargo, si la transformación es local, se tiene:

$\psi(x) \rightarrow \psi^{\prime}(x)=U(\theta) \psi(x)$

donde 
$U(\theta)=\exp \left\{-i \frac{\vec{\tau} \cdot \vec{\theta}(x)}{2}\right\} \psi(x)$

y para la cual (23) no permanece invariante, debido al término

$\bar{\psi}(x) \partial_{\mu} \psi(x) \rightarrow \bar{\psi}^{\prime} \partial_{\mu} \psi^{\prime}=\bar{\psi} \partial_{\mu} \psi+\bar{\psi} U^{-1}(\theta)\left[\partial_{\mu} U(\theta)\right] \psi(x)$

Procediendo como en el caso abeliano, sea $A_{\mu}^{i}$ un conjunto de campos de norma vectorial (uno para cada generador del grupo) y sea la derivada covariante:

$$
\begin{aligned}
& D_{\mu} \psi=\left(\partial_{\mu}-i g \frac{\vec{\tau} \square \vec{A}_{\mu}}{2}\right) \psi \\
& \left(D_{\mu} \psi\right) \rightarrow\left(D_{\mu} \psi\right)^{\prime}=U(\theta) D_{\mu} \psi
\end{aligned}
$$

Entonces usando (30) los campos de norma $A_{\mu}^{i}$ se transforman como:

$\frac{\vec{\tau} \llbracket \vec{A}_{\mu}^{\prime}}{2}=U(\theta) \frac{\vec{\tau} \sqcap \vec{A}_{\mu}}{2} U^{-1}(\theta)-\frac{i}{g}\left[\partial_{\mu} U(\theta)\right] U^{-1}(\theta)$

Para una transformación infinitesimal se tiene de (30) y (24)

$A_{\mu}^{i^{\prime}}=A_{\mu}^{i}+\varepsilon^{i j k} \theta^{j} A^{k}-\frac{1}{g} \partial_{\mu} \theta^{i}$

Donde el segundo término es la transformación para un triplete bajo el grupo SU(2). Entonces los campos de norma $A_{\mu}^{i}$ son portadores de carga, encontraste con el campo de norma abeliano (Yang, et al., 1954).

El término de energía cinética se obtiene del tensor de Faraday dado por:

$F_{\mu \nu}^{i}=\partial_{\mu} A_{v}^{i}-\partial_{v} A_{\mu}^{i}+g \varepsilon^{i j k} A^{j}{ }_{\mu} A^{k}{ }_{\nu}$

El cual se transforma como:

$F_{\mu v}^{i^{\prime}}=F_{\mu v}^{i}+\varepsilon^{i j k} \theta^{j} F_{\mu v}^{k}$

Tal que $F^{i}{ }_{\mu \nu}$ se transforma como un triplete bajo el grupo $S U(2)$.

Sin embargo, el producto $\operatorname{tr}\left(\vec{\tau} \square \vec{F}_{\mu \nu}\right)\left(\vec{\tau} \square \vec{F}_{\mu \nu}\right) \alpha \vec{F}_{\mu \nu} \square \vec{F}_{\mu \nu}$, es invariante bajo está transformación.

El término de masa $-\frac{1}{2} m^{2} \vec{A}_{\mu} \square \vec{A}^{\mu}$ viola la invariancia a menos que los campos de norma tengan masa nula (Goldstone, et al., 1962).

En este caso se tienen términos de tercer y cuarto orden en $\vec{A}_{\mu}$, es decir, se tienen autoacoplamientos entre estos campos (esto se debe a que los $\vec{A}_{\mu}$ tienen carga, no como en el caso 
abeliano). Esto puede generalizarse a cualquier grupo de simetría no abeliana, siguiendo pasos análogos a los vistos anteriormente.

El número de campos de norma coincide con el número de generadores del grupo.

Considerando, como ejemplo a la cromodinámica cuántica (QCD), cuya lagrangiana libre se encuentra dada por (Weinberg, 1967)

$L_{1}=\bar{q}_{j}\left(i \gamma^{\mu} \partial_{\mu}-m\right) q_{j}$

Donde $q_{1}, q_{2}, q_{3}$ denotan los tres colores de quarks. Aquí por simplicidad se considera un solo sabor de quark.

$L_{1}$ es invariante bajo el grupo de transformaciones $S U(3)$.

$q(x) \rightarrow U(x) q(x)=\exp \left\{-i \alpha_{a}(x) t_{a}\right\} q(x)$

Donde $U(x)$ es una matriz unitaria $3 \times 3,\left\{t_{a}\right\}$ con $a=1,2,3, \ldots, 8$ es un conjunto de matrices $3 \times 3$, y los $\alpha_{a}$ son los parámetros del grupo.

En este caso $t_{a}=\frac{\lambda_{a}}{2}$, donde $\lambda_{a}$ son las matrices de GELL-MANN (Halzen, et al., 1984).

La derivada covariante (Pause, et al., 1998) es;

$D_{\mu}=\partial_{\mu}-i g t_{a} G_{\mu}^{a}$

De (32) los campos de norma se transforman:

$G_{\mu}^{a} \rightarrow G_{\mu}^{a}-\frac{1}{g} \partial_{\mu} \alpha_{a}+f_{a b c} \alpha_{b} G_{\mu}^{c}$

Donde $G^{a}{ }_{\mu}$ es el campo del gluón, y su energía cinética está dada por

$$
\begin{aligned}
& L_{0}=-\frac{1}{4} G_{\mu \nu}^{a} G^{a \mu \nu} \\
& G^{a}{ }_{\mu \nu}=\partial_{\mu} G^{a}{ }_{\nu}-\partial_{\nu} G^{a}{ }_{\mu}+g f_{a b c} G^{b}{ }_{\mu} G^{c}{ }_{\nu}
\end{aligned}
$$

La masa del gluón debe ser cero para tener invariancia de norma.

Por lo tanto, la lagrangiana de QCD (cromodinámica cuántica) invariante de norma total es:

$$
L=\bar{q}\left(i \gamma^{\mu} \partial_{\mu}-m\right) q-g\left(\bar{q} \gamma^{\mu} t_{a} q\right) G_{\mu}^{a}-\frac{1}{4} G^{a}{ }_{\mu \nu} G^{a \mu \nu}
$$

La invariancia de norma en $L$ requiere la inclusión (si $m \neq 0$ ) de una auto-interacción entre los bosones de norma cargados.

La ec(41) se puede escribir en forma simbólica que se muestra en la Fig.1:

$L=" \bar{q} q "+" G^{2} "+g " \bar{q} q G "+g " G^{3} "+g^{2} " G^{4} "$ 


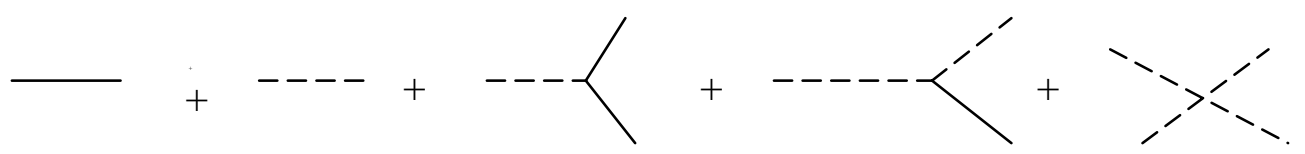

Fig. 1. El grupo de norma no abeliano trae como consecuencia la auto interacción de los campos de norma.

Los tres primeros términos son análogos a QED (electrodinámica cuántica). Estos términos describen la propagación libre de quarks y gluones y la interacción quark-gluón; los dos últimos términos muestran la presencia de vértices con tres y cuatro gluones en QCD, que reflejan el hecho de que los gluones son portadores de carga de color, lo cual no sucede en QED, y esto aparece debido al carácter no abeliano del grupo de norma. Es decir, toda teoría invariante bajo un grupo de norma no-abeliana trae como consecuencia la auto-interacción de los campos de norma.

\section{ROMPIMIENTO ESPONTÁNEO DE SIMETRÍA}

\section{Bosones de Goldstone:}

Hemos visto que en QED y QCD los campos de norma no deben tener masa si la lagrangiana ha de ser invariante. Sin embargo, en interacciones débiles estos campos son masivos $(\approx 80 \mathrm{Gev})$ de hecho el término de masas en la lagragiana introduce divergencias no deseadas ('tHooft, 1971). Así que nuestra tarea es tener campos de norma con masa manteniendo la simetría de $L$.

Cuando se tienen simetrías bajo las cuales $L$ es invariante, pero no así el estado base o vacío, se dice entonces que existe un rompimiento espontáneo de simetría (Abers, et al., 1973). Esto conduce a la generación de masas como se muestra a continuación.

Se tiene a la teoría $\lambda \phi^{4}$ invariante bajo $\phi \rightarrow-\phi$

$L=\frac{1}{2}\left(\partial_{\mu} \phi\right)^{2}-\frac{1}{2} \mu^{2} \phi^{2}-\frac{1}{4} \lambda \phi^{4}$, con $\lambda>0$

Se tiene para $\mu^{2}>0$ y $V=\frac{1}{2} \mu^{2} \phi^{2}+\frac{1}{4} \lambda \phi^{4}$, el vacío corresponde a $\phi=0$.

Si $\mu^{2}<0$, el potencial tiene dos mínimos en:

$\phi= \pm V, \quad V=\sqrt{-\frac{\mu^{2}}{\lambda}}$

Escribiendo a $\phi$ como:

$\phi(x)=V+\eta(x)$

Donde $\eta(x)$ representa las fluctuaciones alrededor del mínimo $V \mathrm{y}$ sustituyendo en la lagrangiana, se obtiene

$L^{\prime}=\frac{1}{2}\left(\partial_{\mu} \eta\right)^{2}-\lambda V^{2} \eta^{2}-\lambda V \eta^{3}-\frac{1}{4} \lambda \eta^{4}$

Tal que $\eta$ adquiere la masa $\sqrt{2 \lambda V^{2}}=\sqrt{-2 \lambda \mu^{2}}>0$. 
Así se genera masa en virtud del rompimiento espontáneo de simetría reflejado en $L^{\prime}$ a través del campo auxiliar $\eta$ (Higgs, 1964).

\section{SIMETRÍA CONTINUA}

Sea $\phi$ un campo complejo definido como; $\phi=\frac{1}{\sqrt{2}}\left(\phi_{1}+i \phi_{2}\right)$ y con una lagrangiana dada por:

$L=\left(\partial_{\mu} \phi\right)^{*}\left(\partial_{\mu} \phi\right)-\mu^{2}\left(\phi^{*} \phi\right)-\lambda\left(\phi^{*} \phi\right)^{2}$

Invariante bajo la transformación $\phi \rightarrow e^{i \alpha} \phi$.

$L=\frac{1}{2}\left(\partial_{\mu} \phi_{1}\right)^{2}+\frac{1}{2}\left(\partial_{\mu} \phi_{2}\right)^{2}-\frac{1}{2} \mu^{2}\left(\phi_{1}^{2}+\phi_{2}^{2}\right)-\frac{1}{4} \lambda\left(\phi_{1}^{2}+\phi^{2}{ }_{2}\right)^{2}$

El mínimo del potencial ocurre en el círculo, Fig.2:

$\phi^{2}{ }_{1}+\phi^{2}{ }_{2}=V^{2}, \quad V^{2}=-\frac{\mu^{2}}{2} ; \quad \lambda>0, \quad \mu^{2}<0$

Como en el caso anterior, sea ahora

$\varphi(x)=\sqrt{\frac{1}{2}}\{V+\eta(x)+i \xi(x)\}$

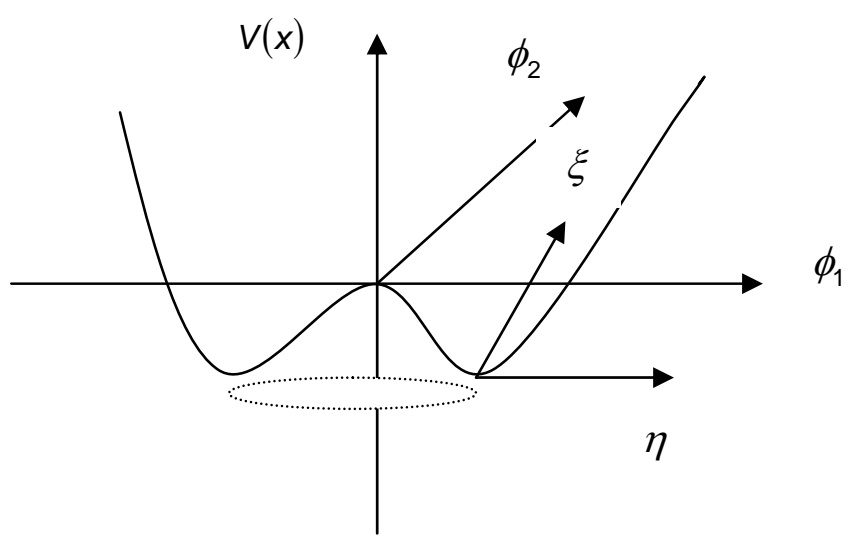

Fig. 2. Cuando se tienen simetrías bajo las cuales $L$ es invariante, pero no así el estado base o vacío, se dice entonces que existe un rompimiento espontáneo de simetría.

Tal que:

$L=\frac{1}{2}\left(\partial_{\mu} \xi\right)^{2}+\frac{1}{2}\left(\partial_{\mu} \eta\right)^{2}+\mu^{2} \eta^{2}+\ldots$

y nuevamente $m_{\eta}=\sqrt{-2 \mu^{2}}$. Pero el campo $\xi$ no tiene masa. Este es un ejemplo del teorema de Goldstone que establece que cuando una simetría interna continua de un sistema se rompe espontáneamente, aparecen partículas escalares sin masa llamados "bosones de Goldstone" (Goldstone, 1961).

Ahora se demostrara este teorema en general, obteniendo también el número de bosones de Goldstone que aparecen en la teoría (Zhang, 2004).

Sea entonces: 
$L=\frac{1}{2} \partial_{\mu} \phi \partial^{\mu} \phi-V(\phi)$

Donde $\phi$ es un vector de $\mathrm{N}$ componentes, $V(\phi)$ es un polinomio en $\phi$ invariante bajo el grupo $\mathrm{G}$, de $\mathrm{N}$ generadores $T_{\alpha}, \mathrm{y} \phi$ se transforma de acuerdo a una representación $\mathrm{N}$-dimensional $L^{\alpha}$ :

$\delta \phi=-i \theta^{\alpha} L^{\alpha} \phi$

Por ser $v(\phi)$ invariante bajo G:

$0=\delta V=\frac{\partial V}{\partial \phi_{i}} \delta \phi_{i}=-i \frac{\partial V}{\partial \phi_{i}} \theta^{\alpha} L^{\alpha}{ }_{i j} \phi_{j}$

Esto es:

$\frac{\partial V}{\partial \phi_{i}} L^{\alpha}{ }_{i j} \phi_{j}=0, \quad \forall \alpha$

diferenciando

$\frac{\partial^{2} V}{\partial \phi_{i} \partial \phi_{k}} L^{\alpha}{ }_{i j} \phi_{j}+\frac{\partial V}{\partial \phi_{i}} L^{\alpha}{ }_{i k}=0$

Evaluando (56) en $\phi=V$, que es el valor de $\phi$ que minimiza al potencial

$\left|\frac{\partial V}{\partial \varphi_{i}}\right|_{\varphi=V}=0$

se obtiene

$\left.\left(\frac{\partial^{2} V}{\partial \varphi_{i} \partial \varphi_{k}}\right)\right|_{\varphi=V} L_{i j}^{\varepsilon} V_{j}=0$

Expandiendo en series de potencias $v(\phi)$ alrededor del mínimo, y conservando sólo los términos lineales:

$V(\phi)=-\frac{1}{2} M^{2}{ }_{i j}(\phi-V)_{i}(\phi-V)_{j}+\ldots$

$\left.\mathrm{y}\left(\frac{\partial^{2} V}{\partial \varphi_{i} \partial \varphi_{k}}\right)\right|_{\varphi=V}=-M^{2}{ }_{i j}$

Donde $M^{2}{ }_{i j}$ es la matriz de masas; así pues se tiene.

$\left(M^{2}\right)_{i j} L_{j k j}^{\varepsilon} V_{k}=0, \quad \forall \alpha$

Sea $S$ un subgrupo de $G$ de $M$ dimensiones y que conserva la simetría del vacío. Si $L^{\alpha}$ es un generador de $S$, entonces $L^{2} V=0, M^{2}{ }_{i j}$ es en general distinta de cero con eigenvalor arbitrario y por lo tanto (61), no contiene información precisa de las masas. Pero para los otros N-M vectores $L^{\varepsilon}{ }_{j k j} V_{k}$ es diferente de cero y (61) nos asegura que $M^{2}{ }_{i j}$ tiene un eigenvalor cero. Si los vectores 
$L^{\alpha} V=0$ están completamente contenidos en un espacio N-M dimensional, entonces existen N-M bosones de Goldstone sin masa en la teoría. Es decir, el número de bosones de Goldstone que van a aparecer en la teoría es igual al número de generadores del grupo que rompen la simetría (Weinber, 1973).

Resumiendo, cuando una simetría discreta se rompe espontáneamente no aparecen los bosones de Goldstone, sin embargo para cuando esto se hace con una simetría continua, entonces si aparecen estos bosones. (Abers, et al., 1973).

\section{MECANISMO DE HIGGS}

Al imponer que una teoría sea invariante bajo transformaciones de norma local, resulta que los campos de norma tienen masa nula, pero al intentar dar masa a estos campos vía un rompimiento espontáneo, por el teorema de Goldstone aparecen en la teoría partículas escalares sin masa (bosones de Goldstone), de tal forma que el problema persiste, ¿Cómo podemos generar masa a los campos de norma sin que aparezcan los bosones de Goldstone? Existe una excepción al teorema de Goldstone, cuando la simetría que se trata sea de norma local, de tal forma que los bosones de Goldstone se combinan con los campos de norma (con la componente longitudinal de polarización) generando campos vectoriales masivos, sin que se pierda el buen comportamiento de la teoría a altas energías (es decir, la teoría es renormalizable, como fue demostrado por 't Hoff en 1971).

A este proceso se le conoce como el mecanismo de Higgs, que se analiza a continuación.

\section{CASO ABELIANO}

Considerando primero el caso más simple de la teoría de norma abeliana, esto es el grupo U(1).

Sea:

$$
L=\left(D_{\mu} \varphi\right)^{\dagger}\left(D_{\mu} \varphi\right)+\mu^{2} \varphi^{\dagger} \varphi-\lambda\left(\varphi^{\dagger} \varphi\right)^{2}-\frac{1}{4} F_{\mu \nu} F^{\mu \nu}
$$

donde

$$
\begin{aligned}
& D_{\mu} \varphi=\left(\partial_{\mu}-i g A_{\mu}\right) \varphi \\
& F_{\mu \nu}=\partial_{\mu} A_{\nu}-\partial_{v} A_{\mu}
\end{aligned}
$$

Se demostró anteriormente que $L$ es invariante bajo la transformación de norma local:

$$
\begin{aligned}
& \varphi(x) \rightarrow \varphi^{\prime}(x)=e^{-i \alpha(x)} \varphi(x) \\
& A_{\mu} \rightarrow A_{\mu}^{\prime}=A_{\mu}-\frac{1}{g} \partial_{\mu} \alpha(x)
\end{aligned}
$$

Cuando $\mu^{2}<0$, el mínimo del potencial

$$
V(x)=-\mu^{2} \varphi^{\dagger} \varphi+\lambda\left(\varphi^{\dagger} \varphi\right)^{2}
$$

ocurre en

$$
|\varphi|=\frac{V}{\sqrt{2}}
$$


donde $V=\left(-\frac{\mu^{2}}{\lambda}\right)^{\frac{1}{2}}$

esto es

$|\langle 0|\varphi| 0\rangle|=\frac{V}{\sqrt{2}}$

Escribiendo a $\phi$ en términos de los campos reales $\phi_{1}, \phi_{2}$

$\phi(x)=\frac{1}{\sqrt{2}}\left(\phi_{1}+i \phi_{2}\right)$

Entonces se puede escoger

$\left\langle 0\left|\varphi_{1}\right| 0\right\rangle=V ; \quad y \quad\left\langle 0\left|\varphi_{2}\right| 0\right\rangle=0$

El mínimo del potencial puede fijar solamente el modulo de $\phi$, de tal forma que existe un número infinito de posibles valores mínimos que se pueden tomar como el vacío físico, lo cual rompe la simetría.

Desplazando los campo

$\phi_{1}^{\prime}=\phi_{1}-V ; \quad \phi_{2}^{\prime}=\phi_{2}$

El campo $\phi_{2}^{\prime}$ corresponde al boson de Goldstone. Ahora al analizar el término de la derivada covariante, se tiene:

$\left|D_{\mu} \phi\right|^{2}=\left|\left(\partial_{\mu}-\mathrm{igA}_{\mu}\right) \phi\right|^{2}=\frac{1}{2}\left(\partial_{\mu} \phi_{1}^{\prime}+\mathrm{gA}_{\mu} \phi_{2}^{\prime}\right)^{2}+\frac{1}{2}\left(\partial_{\mu} \phi_{2}^{\prime}-\mathrm{gA}_{\mu} \phi_{1}^{\prime}\right)^{2}-\mathrm{gAV} \frac{1}{2}\left(\partial_{\mu} \phi_{2}^{\prime}+\mathrm{gA}_{\mu} \phi_{1}^{\prime}\right)+\frac{\mathrm{g}^{2} \mathrm{~V}^{2}}{2} \mathrm{~A}_{\mu} \mathrm{A}^{\mu}$

Donde el último término puede interpretarse como la masa de $A_{\mu}$, el boson de norma adquiere una masa $M=g \vee$. Sin embargo la presencia del término:

$g \vee A^{\mu} \partial_{\mu} \phi_{2}^{\prime}$

el cual mezcla el campo $A_{\mu}$ y $\phi_{2}^{\prime}$, hace que la interpretación no sea clara.

Ahora intententando quitar el término de mezcla (75), para esto se parametriza el campo de la siguiente forma:

$\phi(x)=\frac{1}{\sqrt{2}}[V+\eta(x)] \exp \left\{i \frac{\xi(x)}{V}\right\}=\frac{1}{\sqrt{2}}[V+\eta(x)+i \xi(x)]$

Donde para pequeñas oscilaciones $\eta(x)$ y $\xi(x)$ son $\phi_{1}^{\prime}$ y $\phi_{2}^{\prime}$ respectivamente. En términos de estos campos la lagrangiana libre:

$L_{0}=\frac{1}{2}\left[\left(\partial_{\mu} \eta\right)^{2}-\left(\partial_{\mu} \xi\right)^{2}\right]-\frac{\mu^{2}}{2}\left(\eta^{2}+\xi^{2}\right)$

Ahora para eliminar el término de mezcla (75), se fija la norma, es decir, se utiliza la norma unitaria. 
Para esto se define nuevos campos:

$\phi^{u}(x)=\exp \left\{-\frac{i \xi}{V}\right\} \phi(x)=\frac{1}{\sqrt{2}}(V+\eta(x))$

$B_{\mu}(x)=A_{\mu}(x)-\frac{1}{g V} \partial_{\mu} \xi(x)$

Usando (64) y (65) se obtiene:

$D_{\mu} \varphi=\exp \left\{-\frac{i \xi}{V}\right\}\left(\partial_{\mu} \varphi^{u}-i g B_{\mu} \varphi^{u}\right)=\exp \left\{-\frac{i \xi}{V}\right\}\left(\partial_{\mu} \eta-i g B_{\mu}(V+\eta)\right) \frac{1}{\sqrt{2}}$

entonces:

$\left|D_{\mu} \phi\right|^{2}=\left|\left(\partial_{\mu} \eta-i g B_{\mu}(V+\eta)\right)\right|^{2}$

y también se tiene que

$F_{\mu v}=\partial_{\mu} B_{v}-\partial_{\nu} B_{\mu}$

La lagrangiana (62) toma la forma:

$L=\frac{1}{2}\left|\left(\partial_{\mu} \eta-i g B_{\mu}(V+\eta)\right)\right|^{2}+\frac{\mu^{2}}{2}(V+\eta)^{2}-\frac{\lambda}{4}(V+\eta)^{4}-\frac{1}{4}\left(\partial_{\mu} B_{v}-\partial_{\nu} B_{\mu}\right)^{2}=L_{0}+L_{i}$

Donde

$L_{0}=\frac{1}{2}\left(\partial_{\mu} \eta\right)^{2}-\frac{1}{2} \mu^{2} \eta^{2}-\frac{1}{4}\left(\partial_{\mu} B_{v}-\partial_{\nu} B_{\mu}\right)^{2}+\frac{1}{2}(g V)^{2} B_{\mu} B^{\mu}$

$L_{i}=\frac{1}{2} g^{2} B_{\mu} B^{\mu} \eta(2 V+\eta)-\lambda V^{2} \eta^{3}-\frac{1}{4} \lambda \eta^{4}$

De aquí se observa, que se tiene en la teoría un boson vectorial masivo con masa $M=g V$, un mesón escalar $\eta$ con masa $m_{\eta}=\sqrt{2} \mu$. El campo $\xi(x)$ desaparece de la lagrangiana. Ahora contando los grados de libertad antes y después del rompimiento espontáneo de simetría: Antes se tenían dos campos escalares $\phi_{1}$ y $\phi_{2}$, un boson de norma sin masa $A_{\mu}$ con dos grados de polarización; después del rompimiento se tiene; un campo escalar masivo $\eta$ y un boson de norma masivo $B_{\mu}$ el cual tiene tres estados de polarización. Entonces, el boson de Goldstone $\xi(x)$ se combina con el tercer estado de polarización produciendo un campo vectorial masivo $B_{\mu}$. A este proceso se le conoce como el mecanismo de Higgs (Higgs, 1966).

La generalización al caso no - abeliano es similar.

Estas ideas se aplican en el modelo de Weinberg - Salam, la cual es una teoría que unifica a las interacciones débiles y electromagnéticas, usando como grupo de norma $S U(2) \times U(1)$ con rompimiento espontáneo de simetría (Weinberg, 1973, Salam, 1968). 


\section{CONCLUSIONES}

La invariancia de norma local $U(1)$, por ejemplo, implica la invariancia global $U(1)$, pero el reciproco no es cierto. Una masa pequeña para el fotón destruiría la invariancia de norma de la electrodinámica, pero no afectaría sus efectos microscópicos, incluyendo algunos cuánticos, como por ejemplo la conservación de la carga eléctrica. En particular tal teoría sería también renormalizable puesto que los fotones polarizados longitudinalmente se desacoplan de la corriente conservada.

Sin embargo las simetrías globales no son buenos candidatos para fundamentar primeros principios. Una simetría global nos da libertad de convención de escoger un sistema de referencia, tal como lo establece Carvalho (Carvalho, 2004).

Las teorías de norma local, nos dan una descripción consistente de las leyes de la naturaleza basándose en principios bien establecidos, los cuales han sido fenomenológicamente probados. Hoy en día se sabe, que esto es válido no sólo para la electrodinámica con su simetría de norma abeliana $U(1)$, sino también para las interacciones débiles y fuertes, descritas por teorías de campos de norma con grupos de simetría no - abeliana: $S U(2) x U(1)$ y $S U(3)$ respectivamente. Las simetrías de norma no - abeliana son más restrictivas y más profundas que la simetría $U(1)$. En particular los bosones de norma no - abeliana son portadores de las cargas del grupo y sus términos de masa en la lagrangiana en general a menos que se introduzcan por medio de un rompimiento espontáneo de simetría, destruyen no sólo la simetría de norma sino también la conservación de la corriente y por lo tanto la renormalizabilidad de la teoría.

\section{REFERENCIAS}

Abers, E.S., Lee, B. W. Physics Reports C, 9, 1-141, (1973).

Bjorken, J. Drell, S. Relativistic Quantum Fields, ed. Mc Graw Hill, (1986).

Bogoliubov, N, Shirkov, D.V. Introduction to the theory of Quantized Fields. New York, Interscience Publishers, Inc. (1959).

Brihaye, Y., Hartmann, B., Radu, E. Boson Star in SU(2) Yang - Mills scalar field theories. Physics letters B. 607, 17-26, (2005)

Carvalho, M. Generalized BRST models and topological Yang - Mills theories. J. Geometry and Phys. 51, 40-70 (2004)

Cabibbo, N., Phys Rev. Letters 10, 531-538, (1963).

Catren, G., Geometric foundations of classical Yang-Mills theory. Modern Phys. 39, 511-531 (2008)

Damour, T., Nicolai, H. Symmetries, singularities and the demergence of space. International Journal of modern Phys. D 17, 525-531, (2008)

Faddeev, L.D., Popov, V.N., Phys. Letters B, 25, 29-42 (1967).

Feynman, R., Phys. Rev. 80, 440-485, (1950).

Feynman, R. P. Hibbs, A. R. Quantum Mechanics and Path Integrals, Mc Graw Hill, (1965).

Georgi, H., Weak Interactions and Modern Particle Theory, Dover Publications. (2009) 
Gibson, W. M., Pollar, B. R. Symmetry Principles in Elementary Particle Physics, Cambridge University Press. (1976).

Goldstone, J.. Nuovo Cimento 19, 15-42, (1961).

Goldstone,J., Salam, A., Phys. Rev. 127, 965-985, (1962).

Halzen, F. Martin, A. Quarks and Leptons. An Introductory Course in Modern Particle Physics. John Wiley and Sons. (1984).

Harikumar, E., Mitra, I., Sharatchandra, H.S. Topological fields paterns of the Yang-Mills theory. Phys. Letters B557, 297-302 (2003)

Higgs, P.W., Phys. Rev. 12, 132-141, (1964).

Higgs, P.W., Phys. Rev. Letters 145, 1156-1181, (1966).

Itzykson, C., Zuber, J.B. Quantum Field Theory, Dover Publications Inc. 2005

Kiefer, C. Quantum Gravity, second edition, Oxford University Press, Oxford, 2007

Leite L., J. Introducción a la Electrodinámica Cuántica. Ed. Trillas. (1977).

Pause, T. Heinzl, T., The configuration space of low-dimensional Yang-Mills theory. Nuclear Phys. B524, 695-741 (1998).

Salam, A., Elementary particle theory. Ed. N. Svartholm (Almquist an Forlag, Stockolm), (1968).

Schwinger, J. Quantum Electrodynamics. Ed. Dover Publications, (1987).

Schweber, S. An Introduction to Relativistic Quantum Field Theory. Ed. Dover Publications, Inc.. (2005).

Taylor, J. C. Gauge Theories of Weak Interactions. Ed. Cambridge University Press, (1976).

Vacaru, S. Exact solutions with nocommutative symmetries in Einstein and gauge gravity. J. Math. Phys. 46, 042503 (2005)

Weinberg, S., Phys. Rev. Letters 19, 1264-1290, (1967).

Wienberg, S., General theory of broken local symmetries, Phys. Rev. D7, 1068, (1973).

Weyl, H., The Theory of Groups and Quantum Mechanics. Ed. Dover Publications, Inc. (1950).

Xi Zhang. Compactness theorems for coupled Yang - Mills fields. J. Math. Anal. Appl. 298, 261278 (2004)

Yang, C.N., Mills, R., Phys. Rev. 96, 191-221, (1954).

'tHooft, G., Emergent quantum mechanics and emergent symmetries, presented at PASCO B, Imperial College, London. July 6, 2007.

'tHooft, G., Nucl. Phys. B, 35, 167-181, (1971). 
